\title{
Eye and hand movements during reconstruction of spatial memory
}

\author{
Melanie R Burke, Richard J Allen, Claudia Gonzalez \\ Institute of Psychological Sciences, University of Leeds, Leeds LS2 9JT, UK; \\ e-mail: m.r.burke@leeds.ac.uk \\ Received 19 January 2012, in revised form 13 June 2012
}

\begin{abstract}
Recent behavioural and biological evidence indicates common mechanisms serving working memory and attention (eg Awh et al, 2006 Neuroscience 139 201-208). This study explored the role of spatial attention and visual search in an adapted Corsi spatial memory task. Eye movements and touch responses were recorded from participants who recalled locations (signalled by colour or shape change) from an array presented either simultaneously or sequentially. The time delay between target presentation and recall $(0,5$, or $10 \mathrm{~s})$ and the number of locations to be remembered (2-5) were also manipulated. Analysis of the response phase revealed subjects were less accurate (touch data) and fixated longer (eye data) when responding to sequentially presented targets suggesting higher cognitive effort. Fixation duration on target at recall was also influenced by whether spatial location was initially signalled by colour or shape change. Finally, we found that the sequence tasks encouraged longer fixations on the signalled targets than simultaneous viewing during encoding, but no difference was observed during recall. We conclude that the attentional manipulations (colour/shape) mainly affected the eye movement parameters, whereas the memory manipulation (sequential versus simultaneous, number of items) mainly affected the performance of the hand during recall, and thus the latter is more important for ascertaining if an item is remembered or forgotten. In summary, the nature of the stimuli that is used and how it is presented play key roles in determining subject performance and behaviour during spatial memory tasks.
\end{abstract}

Keywords: attention, memory, motor behaviour, recall, vision

\section{Introduction}

Working memory has been defined as a multi-component, limited-capacity, short-term memory storage system capable of actively maintaining and manipulating a range of information to guide goal-directed behaviour (Baddeley and Hitch 1974). It is a fundamental precursor to higher cognitive functions such as learning and language comprehension (Baddeley 2003) and constitutes a set of processes essential for normal daily functioning. Agreement within theories of working memory processing comes down to the basic processing stages, specifically, encoding, maintenance, and retrieval (Shah and Miyake 1999), with a consensus regarding the specific construct of working memory yet to be reached (Cowan 2001).

An important cognitive ability attributed to working memory concerns the temporary storage and processing of spatial information - that is, the provision of a consciously accessible online record of where in the environment items were located. According to Baddeley's influential model of working memory (eg Baddeley 1986,2003; Baddeley and Hitch 1974), spatial memory is one of the main functions of the visual-spatial sketchpad sub-component. However, it has been argued that visual and spatial processing (the what and the where) should be fractionated into further connected but separable sub-components (eg Della Sala et al 1999; Klauer and Zhao 2004; Logie 1995; Tresch et al 1993), with these processes possibly based within different areas of the brain (eg Courtney et al 1996; Müller and Knight 2006). For example, Della Sala et al (1999) observed that a concurrent spatial interference task showed greater disruption of performance on a spatial memory task (Corsi span), relative to the minimal effects of a concurrent visual task (which in turn disrupted 
a task measuring visual short-term memory). The Corsi task (Corsi 1972; De Renzi and Nichelli 1975; Grossi et al 1993; Milner 1971) is a commonly used method of assessing spatial memory. In this measure, a sequence of spatial locations is highlighted, before participants attempt to reproduce this sequence themselves. This task has traditionally been implemented with wooden blocks fixed to a board, with the experimenter and participant signalling each block using motor movements. More recently, computer-based adaptations of the task have also been developed and implemented in experimental (eg Pearson and Sahraie 2003; Saint-Aubin et al 2007; Smyth and Scholey 1994; Vandierendonck et al 2004) and clinical (eg Joyce and Robbins 1991) settings.

An important element in encoding and retaining spatial information in working memory is the allocation of spatial attention and the role of eye movements in these processes. For example, Saint-Aubin et al (2007) demonstrated that recall accuracy for sequentially presented dot locations showed a positive relationship with fixation duration during encoding. In line with this, it has been found that performing ocular suppression (through the guiding of participants' eye movements) during encoding in this task disrupts recall (Guérard et al 2009). Similar processes may be important in keeping spatial representations active once they are retained in working memory. Work by Awh and colleagues (eg Awh and Jonides 2001; Awh et al 1998, 2006a) suggests common mechanisms and neural circuitry underlie spatial attention and spatial working memory. In particular, Awh et al (1998) proposed an attention-based rehearsal hypothesis, in which spatial information is actively maintained via the shifting of spatial attention to locations retained in working memory (through interactions within a frontal-parietal-occipital network). In line with this, Smyth and Scholey (1994; Smyth 1996) found that imposing covert shifts in spatial attention disrupted memory for spatial locations. Similarly, Awh and Jonides (2001) demonstrated that when a location is maintained in working memory, visual attention processing at the memorised location is facilitated, just as attending to a location improves the ability to remember information at that location (Posner 1980).

Though this viewpoint has received much support, more recent studies challenge Awh et al's (1998) hypothesis, indicating that the role of attention in spatial working memory may not be as central as previously considered. For example, Pearson and Sahraie (2003) found that shifts in eye movements during retention had larger disruptive effects than did changes in covert spatial attention (see also Belopolsky and Theeuwes 2009; Chan et al 2009; Guérard et al 2009; Theeuwes et al 2009; Tremblay et al 2006 for the importance of eye movements in retaining spatial information). These findings have instead been interpreted as indicating that an oculomotor control network is influential in rehearsing location information in working memory. In line with this, Curtis (2006) suggested that spatial attention and spatial working memory might be based on activation within the frontal eye field.

It is therefore clear that eye movements play a key role in encoding and retaining spatial information. However, this theoretical and methodological approach has to date been limited to explorations of the processes occurring during the encoding and/or retention phases of spatial memory tasks. To our knowledge, no previous studies have examined eye movement and touch responses during the reconstruction of spatial displays. This is surprising, given that the response phase is a crucial element of spatial memory tasks. The non-redundancy of eye movement and recall data in assessing memory processes (Saint-Aubin et al 2007) clearly underlines the need for such an exploration, in order to help develop a clearer understanding of spatial memory tasks.

The present experimental work therefore implemented computer-based adaptations of the Corsi spatial memory task, in which a set of spatial locations were cued during presentation, with participants required to reproduce this display at the recall phase using 
responses to a touch screen. Importantly, we recorded eye movement information during this reconstruction phase. Furthermore, we manipulated a number of factors in order to gauge how they affect response accuracy/speed and eye movements. Recently, Zhang et al (2010) showed behavioural and electrophysiological evidence of the effects of stimulus attributes in working memory and how these guide attention. Results showed that high complexity stimuli are less effective in guiding attention compared to attributes such as colour that are easily held in working memory (Zhang et al 2010). As with EEG measures, eye movements may be able to provide information of attentional strategies used in working memory tasks.

\subsection{Simultaneous versus sequential presentation}

The first factor we manipulated was whether spatial locations were cued simultaneously or in a sequential fashion. Visuospatial memory has previously been shown to be relatively less accurate when presentation is sequential (eg Allen et al 2006; Blalock and Clegg 2010; Frick 1985; Igel and Harvey 1991; Lecerf and de Ribaupierre 2005; Zimmer et al 2003). A number of explanations have been proposed, including retroactive interference impacting on representations of sequentially presented items or simultaneously encountered arrays benefiting from the encoding of global patterns as well as individual item information.

It is important to note that sequential presentation also involves processing of temporal order. Order memory may have an executive-attention element, and may possibly be mediated by areas within the prefrontal cortex (D'Esposito et al 1995), particularly when intentional encoding of order is important (eg Mangels 1997; Marshuetz et al 2000; Sakai and Passingham 2003). We would predict a greater number of eye movements and longer fixation times due to a higher cognitive load during sequential presentations, relative to order-free simultaneous conditions. A primary aim of this study was therefore to examine how eye movements might vary following sequential presentation, when order information is important, versus simultaneous presentation, and what this tells us about reconstruction processes in each case.

\subsection{Change salience: colour versus shape}

Most forms of computerised Corsi task or equivalent typically signal spatial locations using changes in colour. In this study, we manipulated the type of feature change used to signal locations, specifically comparing colour change and shape change trials. The visual attention literature indicates that colour is processed faster than shape and is less cognitively demanding (Eimer 1997; Proverbio et al 2004). Feature-integration theory (Treisman and Gelade 1980) was the first influential theory of attention to suggest that features such as shape and colour are distinctive in that they exist in separate maps in pre-attentive vision. It has also been suggested that colour processing holds a special status in attention selection compared to other non-spatial features including shape (Proverbio et al 2004).

The colours used in the present study were visually salient primary colours (red and blue) that have been suggested to be especially resistant to memory decay (Uchikawa and Shinoda 1996). In contrast, simple geometric shapes were used with a low change salience, specifically squares to circles, for the low-attention condition. Salience is an important determinant of attentional allocation (eg Fecteau and Munoz 2006; Itti and Koch 2001; Koch and Ullman 1985). If a common oculomotor system underlies spatial attention and working memory (eg Curtis 2006; Pearson and Sahraie 2003), we might then expect similar effects of variables on tasks tapping each of these elements. In line with this, it has been demonstrated that where spatial attention is allocated influences later memory accuracy (eg Schmidt et al 2002). Hence, this study aimed to identify differences in performance between shape and colour, with the hypothesis that performance would be better on colour change trials due to the greater salience of these changes during encoding. We also expected to observe concomitant changes in eye movement. 


\subsection{Delay}

A further manipulation implemented in this study was that of a varying delay (specifically, $0 \mathrm{~s}, 5 \mathrm{~s}$, or $10 \mathrm{~s}$ ) between the presentation of stimuli and recall of the targets. Studies have suggested that qualitatively different processes take place for short delays (around $1 \mathrm{~s}$ ) compared to longer delays (around $6 \mathrm{~s}$ ) in visual working memory tasks (Baddeley 1986; Phillips 1974; Treisman and Zhang 2006). For example, Phillips (1974) found that visual pattern memory declined over delays of up to $9 \mathrm{~s}$ (although Vogel et al 2001 did not observe any accuracy decrement over delays of $1-5 \mathrm{~s}$ ). One process that occurs during retention is consolidation, whereby items are transformed from a perceptual format into more durable working memory representations, thought to occur between around $200 \mathrm{~ms}$ and $1 \mathrm{~s}$, following presentation of the stimuli (eg Treisman and Zhang 2006). This duration is thought to increase with the number of targets, indicating that consolidation is a time-consuming and limitedcapacity process (Vogel et al 2006). Furthermore, as delay increases so does the threat of information loss through decay or interference. This threat may increase the demand for attentional resources in keeping representations active, thus the length of delay may define the level of involvement of the underlying neural mechanisms involved in attention and working memory (Elliot and Dolan 1999). In line with this, while Dreher et al (2001) did not find significant differences between $500 \mathrm{~ms}$ and $10 \mathrm{~s}$ delays in healthy controls using a set of spatial memory reconstruction tasks very similar to those in the current study, they did find large delay effects in schizophrenic patients. On the basis of Dreher et al's (2001) findings, we predicted no effect of delay on accuracy in our own sample of healthy adult participants. However, as with the other manipulated variables, our main focus (for which we had no a priori prediction in this case) was on eye movement data during reconstruction.

\subsection{Set size}

The final variable implemented was the number of targets presented in a trial. As noted, working memory is a limited capacity system (Baddeley and Hitch 1974) and research suggests the capacity of visual working memory is such that only three to four objects can be maintained simultaneously (eg Cowan 2001; Luck and Vogel 1997; Vogel et al 2001). In terms of tasks that are closely analogous to our paradigm, healthy adults typically achieve a Corsi span score of around 5 locations. As our primary focus was concerned with how eye movement patterns might vary under sub-span and span conditions, we implemented set sizes between 2 and 5 items in this experiment. Dreher et al (2001) found accuracy decrements from set sizes 2-5 in healthy adults and schizophrenic patients. Similarly, Magen et al (2009) observed a linear increase in reaction time as a function of load $(1,3,5$, or 7$)$, supporting findings from previous studies (Linden et al 2003; Todd and Marois 2005). We therefore predicted longer touch reaction times and more response errors with an increasing number of targets.

Of greater interest in terms of the novelty of our study was whether eye movement patterns would also vary with increasing set size. We propose that eye movements would mimic the hand responses, in that subjects fixate longer on targets with increasing set size due to increased cognitive demand particularly during the encoding phase of the response. Alongside this hypothesis we would also suggest that sequential presentation of the data encourages longer fixations during encoding, due to the more salient nature of a single target to fixate.

As far as we are aware, this study represents the first exploration of eye and hand movements during reconstruction of spatial working memory and how they may be influenced by different factors (ie attention, delay, and memory load). This experiment therefore provides novel insights into the processes operating during the reconstruction phase, with implications both for models of spatial working memory and for the processes underlying different variants of the Corsi task, a commonly used measure of individual differences in spatial working memory ability. 


\section{Method}

\subsection{Participants}

A sample of twenty healthy young adults (ten males and ten females), recruited through the University of Leeds, participated in the study. Participants were aged between 20 and 32 years, with no known neurological disorders or colour-blindness. All were right-handed and had normal or corrected-to-normal vision. All participants provided informed consent prior to commencing the study.

\subsection{Materials}

Participants were seated in a dark room with a distance of $38 \mathrm{~cm}$ from the monitor screen to the eye. Subjects' touch responses were recorded with a MagicTouch USB touch screen (Keytec Ltd) which was fixed to a CRT 19 in monitor. An EyeLink 1000 (SR Research Ltd) tower-mounted eye-tracking system was used to record participants' eye movements throughout the experiment at $1000 \mathrm{~Hz}$. The participants were seated with their chin and forehead supported by padded rests to keep head movements to a minimum during the experiment. Experiment Builder software was used to generate stimuli while DataViewer was used to save and analyse the data (SR Research Ltd) alongside custom made Matlab programmes (The MathWorks, Natick, MA).

\subsection{Stimuli and design}

Twelve blue squares, each 60 pixels $\times 60$ pixels, were present on a black screen in each trial (figure 1). Participants were presented with two stimulus change conditions (colour and

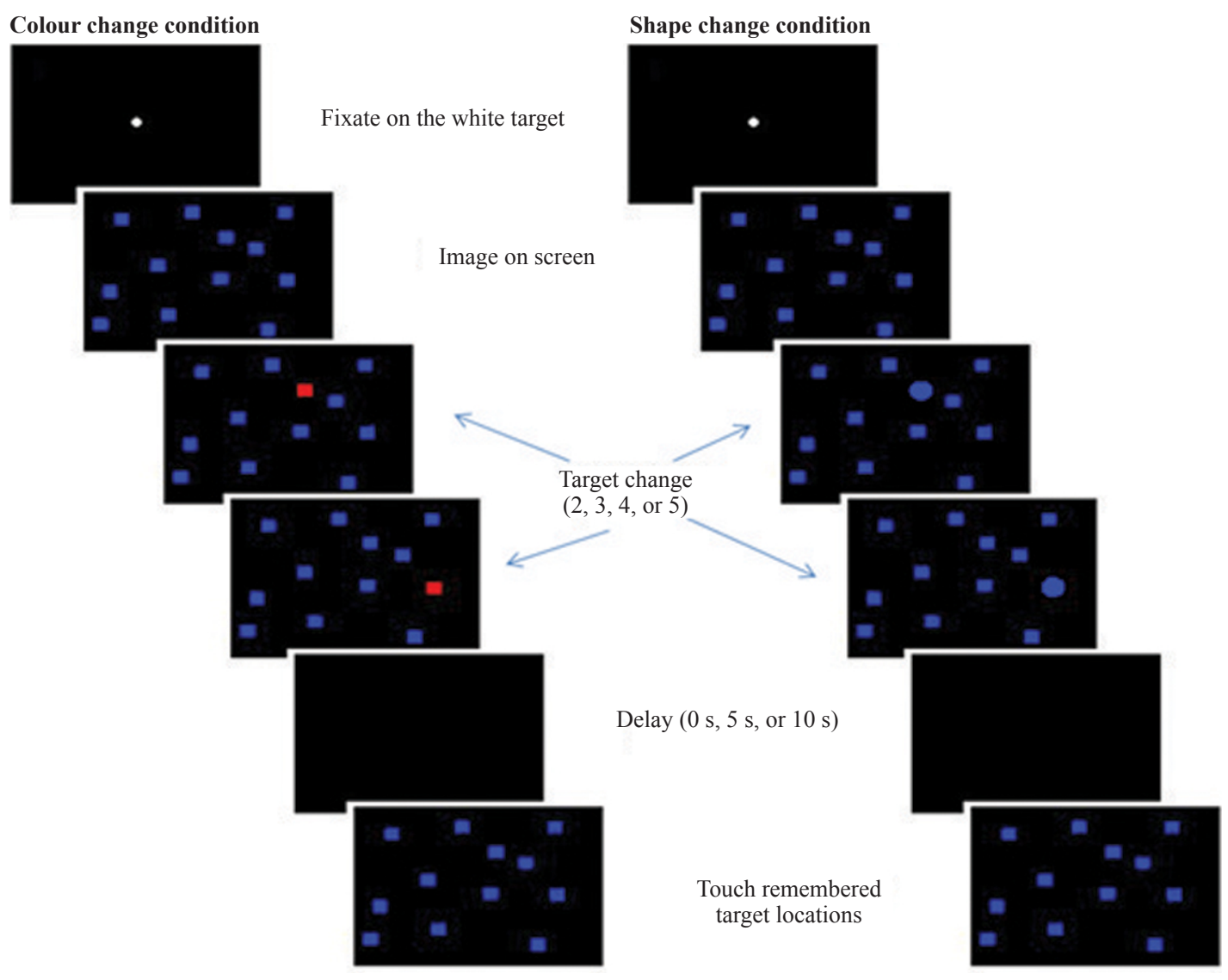

Figure 1. [In colour online, see http://dx.doi.org/10.1068/p7216] A diagram to illustrate the procedure. Participants were required to remember either the sequence of target change or just the location. The first 4 screens (before the delay) show the acquisition phase and the final screen of the response phase of the trial. 
shape condition) and two types of target presentations in each condition (simultaneous target change and sequential target change $-\mathrm{S}, \mathrm{C}, \mathrm{SC}$, and $\mathrm{CC}$ ), resulting in a total of 4 blocks from these combinations (1a, 1b, 2a, and 2b). Each block comprised 36 trials (144 trials, per subject, per experimental session), in which the delay between acquisition and recall $(0,5$, or $10 \mathrm{~s})$ and the number of targets to recall (between 2 and 5) was also varied. This resulted in 12 repetitions of each delay and 9 repetitions of each set size per block, which was randomly intermixed. In the colour condition (1a and $1 \mathrm{~b}$ ) squares changed from blue to red, an obvious change requiring low attention, while in the shape condition ( $2 \mathrm{a}$ and $2 \mathrm{~b}$ ) the target squares changed to circles, a less salient difference possibly demanding higher levels of attention (please see figure 1). The sequential target change condition required participants to remember the target changes in the exact order presented, while the simultaneous condition allowed the participant to recall the target change locations in any order. These blocks were counterbalanced between participants to eliminate order effects. Each stimulus was presented for $1 \mathrm{~s}$ - ie in sequential trials with five targets, each of the five targets were presented for $1 \mathrm{~s}$ each, or in simultaneous trials all five targets were visible for $5 \mathrm{~s}$.

\subsection{Procedure}

All participants completed two practice trials prior to each of the four blocks (1a, 1b, 2a, and $2 b)$ of 36 trials. They were given short breaks in between trial sets to reduce the effects of fatigue and dark adaptation. The presentation phase in each trial involved 2-5 of the 12 targets changing colour or shape, either simultaneously (no sequence) or one by one in a particular order (sequence). Following this change the screen went blank for a variable amount of time $(0 \mathrm{~s}, 5 \mathrm{~s}$, or $10 \mathrm{~s})$. The 12 original targets would then appear, and participants were required to either touch the location of the targets in any order (in simultaneous presentation conditions) or touch the location of the targets in the correct order (in sequential presentation conditions). Touch responses needed to be within the boundaries of a blue square for the programme to accept it as a true response, with a "beep" signalling to the participant that a response has been recorded. All touch data were recorded, and errors were collated and counted a posteriori.

\subsection{Data analysis}

We obtained the following parameters for each of these conditions: (i) touch time (reaction time between each touch response per trial), (ii) touch accuracy (number of incorrect touches per trial), (iii) fixation duration (mean fixation duration on each item per trial), and (iv) saccade amplitude (mean saccade distance between each saccade, excluding saccades $<1 \mathrm{deg}$, within a trial). The mean for each subject during the response/recall phase of the trial was obtained for each (i) feature (colour or shape), (ii) presentation type (simultaneous or sequential), (iii) delay (0 ms, $5000 \mathrm{~ms}$, and $10000 \mathrm{~ms}$ ), and (iv) set size (number of targets - 2, 3, 4, or 5). A series of 2 (feature: colour and shape) $\times 2$ (target change: simultaneous and sequential) $\times 2$ (delays: no delay versus delay) $\times 4$ (set size: $2-5$ targets) repeated-measures ANOVA were used to analyse touch and eye data. Interactions between variables were evaluated with Bonferroni-corrected a posteriori test. A significance level of $p<0.05$ was established for all statistical analyses. Results are expressed as means \pm standard error.

Furthermore we performed a region of interest (ROI) analysis that recorded the time the eye spent within the boundaries of a signalled target that either changed colour or shape during the trial (ie a relevant/correct target). These data were segregated for (i) the encoding phase, (ii) the delay phase, and (iii) the recall phase for each trial and for each condition. We then calculated the time spent within the ROIs for each phase of the trial and then divided this by the total time of that phase. The results were converted into a percentage of time the eye spent on relevant target items within each phase (\% dwell time); means and standard deviations were obtained for each subject and significance found through a repeated-measures ANOVA. 


\section{Results}

Touch accuracy and touch time will be reported first, before eye fixation duration and saccade amplitude. A series of analyses comparing $5 \mathrm{~s}$ and $10 \mathrm{~s}$ delay conditions on each measure failed to reveal any significant main effects or interactions ( $p>0.05$ in all cases). Therefore, in order to simplify the analyses, $5 \mathrm{~s}$ and $10 \mathrm{~s}$ delay trials were collapsed together and treated as a single "delay" condition (in comparison with "no delay") for all subsequent analyses.

\subsection{Touch results}

Mean touch time and standard error (in $\mathrm{ms}$ ) for each experimental condition is displayed in figure 2 . A $2 \times 2 \times 2 \times 4$ repeated-measures ANOVA was performed, examining the effects of presentation type (sequential versus simultaneous), feature salience (colour versus shape), delay (no delay versus delay), and set size $(2,3,4,5$ locations). This revealed non-significant effects of presentation type $\left(F_{1,19}=3.36, p=0.08\right)$ and feature salience $\left(F_{1,19}=0.35, p=0.56\right)$, but a significant increase in touch time with a delay $\left(F_{1,19}=12.68\right.$, $p<0.01)$ and a significant difference in set size $\left(F_{3,57}=3.98, p<0.05\right)$ was observed. More specifically, we found a difference between 2 and 3 targets $(p=0.011)$ and 2 and 4 targets $(p=0.005)$, but not between 2 and 5 targets $(p=0.240)$. There were no significant interactions $(p>0.05)$.
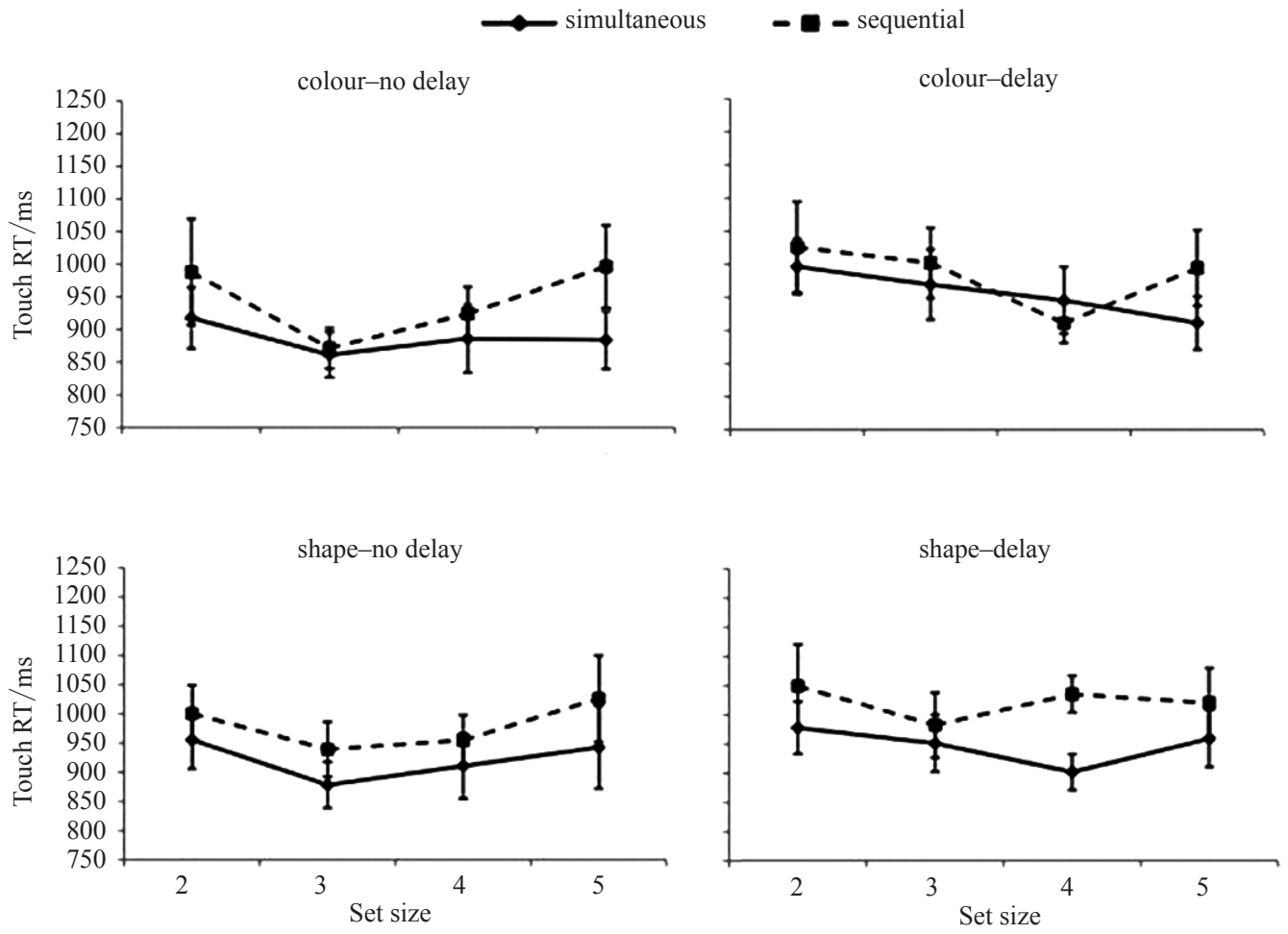

Figure 2. Mean reaction time (ms) and standard error for touch responses as a function of presentation format, feature change, delay, and set size.

Error rates were calculated as the proportion of errors made for each individual touch response in each condition. This measure controls for the greater number of possible errors on trials with larger set sizes. Mean proportional error rate and standard error are displayed in figure 3. A $2 \times 2 \times 2 \times 4$ repeated-measures ANOVA revealed significant effects of presentation type $\left(F_{1,19}=8.68, p<0.05\right)$, with less errors for simultaneously presented stimuli and an increasing number of errors with increasing set size $\left(F_{3,57}=3.91, p<0.05\right)$. In contrast, there was no effect of feature salience $\left(F_{1,19}=2.04, p=0.17\right)$ or delay $\left(F_{1,19}=1.99, p=0.18\right)$. 


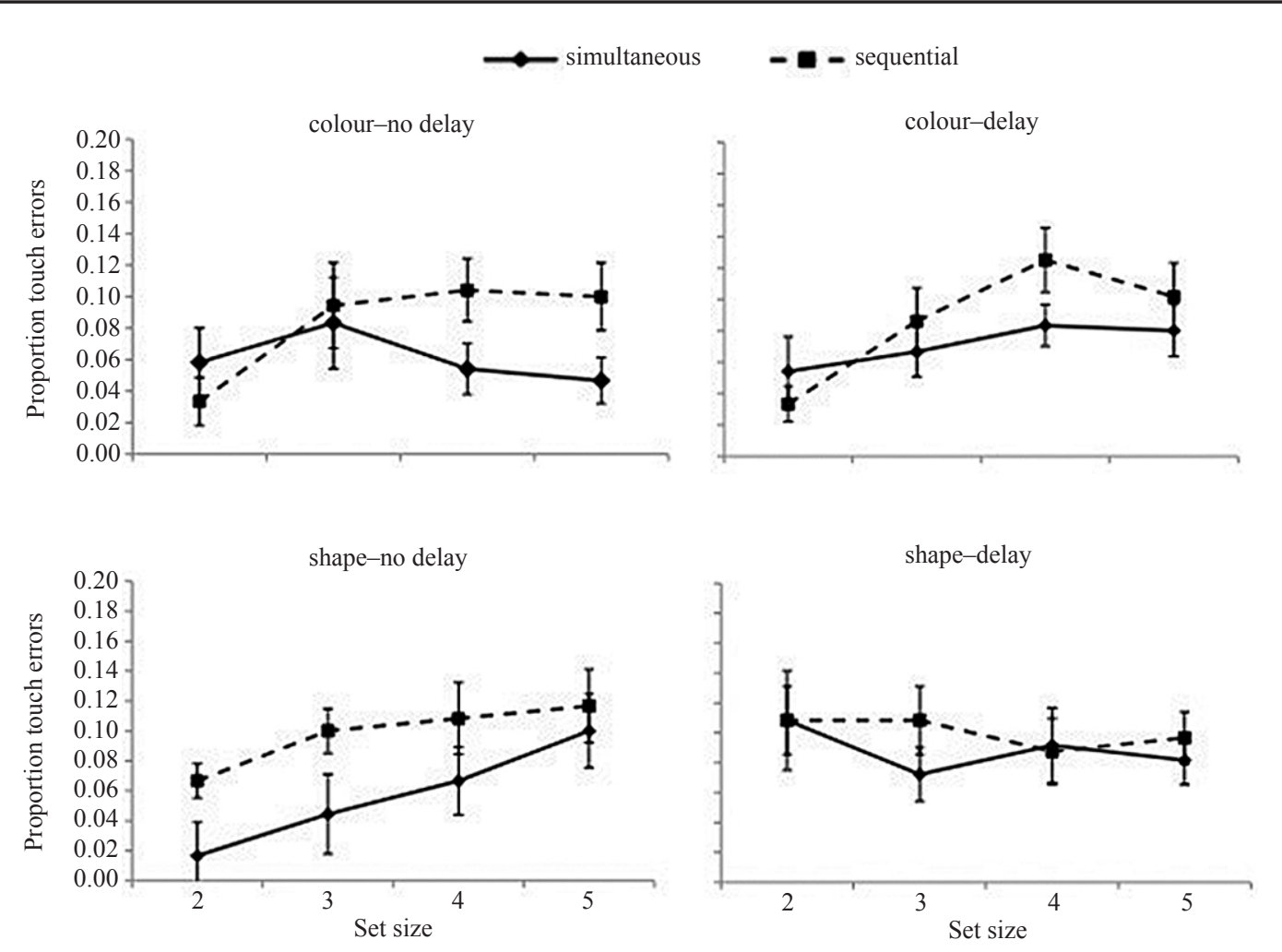

Figure 3. Mean proportional error rate and standard error for touch responses as a function of presentation format, feature change, delay, and set size.

\subsection{Eye movement results}

Mean eye-fixation duration and standard error (in $\mathrm{ms}$ ) is illustrated in figure 4. The analysis revealed significant effects of presentation type $\left(F_{1,19}=10.86, p<0.01\right)$ and feature salience $\left(F_{1,19}=13.29, p<0.01\right)$. Fixation durations were longer during sequential presentation trials and trials involving colour changes. However, the effects of delay $\left(F_{1,19}=0.24, p=0.63\right)$ and set size $\left(F_{3,57}=0.95, p=0.40\right)$ were not significant. We also found a significant two-way interaction between feature salience and delay $\left(F_{1,19}=8.24\right.$, $p<0.05)$, with longer fixation durations for colour than shape trials only apparent after no delay and not after a delay.

Mean saccade amplitude (in degrees of visual angle) during a trial and standard error are displayed in figure 5. There were no significant effects of feature salience $\left(F_{1,19}=0.98\right.$, $p=0.34)$, presentation type $\left(F_{1,19}=1.40, p=0.25\right)$, or delay $\left(F_{1,19}=2.05, p=0.17\right)$, while the effect of set size was marginally non-significant $\left(F_{3,57}=2.74, p=0.052\right)$.

In order to investigate the effect of fixation duration on the resultant accuracy and timing of the response, we calculated mean dwell time (ROI analysis) for all subjects during (i) the encoding phase of the trial and (ii) the recall phase of the trial (see figure 6). We found a significant effect of presentation type $\left(F_{3,17}=14.287, p<0.001\right)$, delay $\left(F_{2,18}=5.751\right.$, $p<0.05)$, and set size $\left(F_{3,17}=25.444, p<0.001\right)$. Pairwise comparisons revealed that during encoding the sequentially presented stimuli showed a significantly higher percentage of dwell time within a selected target than the simultaneous stimuli for both colour $(p<0.001)$ and shape $(p<0.001)$. Furthermore, an interaction between presentation type and set size was also found $\left(F_{9,11}=7.696, p<0.01\right)$, particularly between 2 and $4(p<0.01)$ and 2 and 5 items $(p<0.001)$. We found no significant effects or interactions during the delay or recall phases of the trial. 


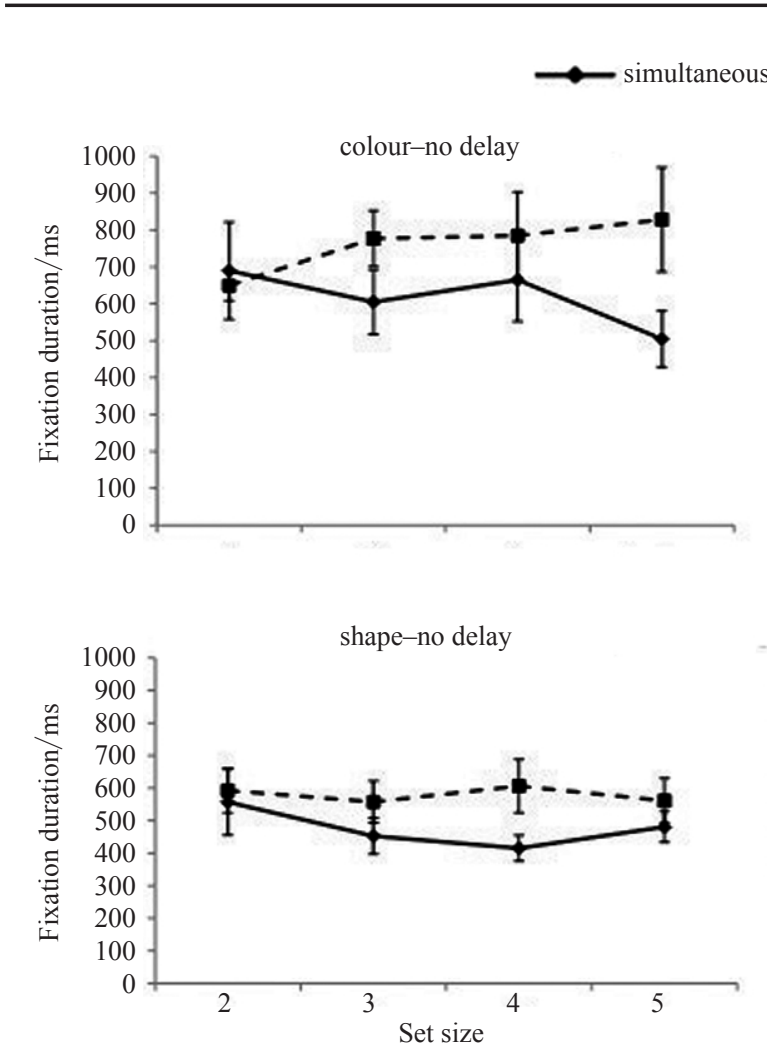

- - sequential
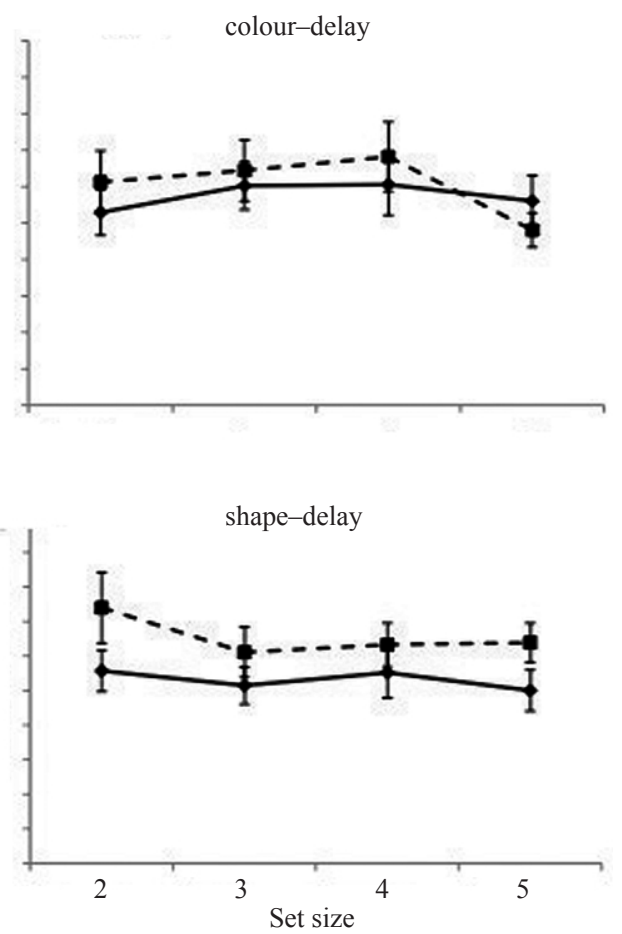

Figure 4. Mean fixation duration (ms) and standard error during reconstruction as a function of presentation format, feature change, delay, and set size.
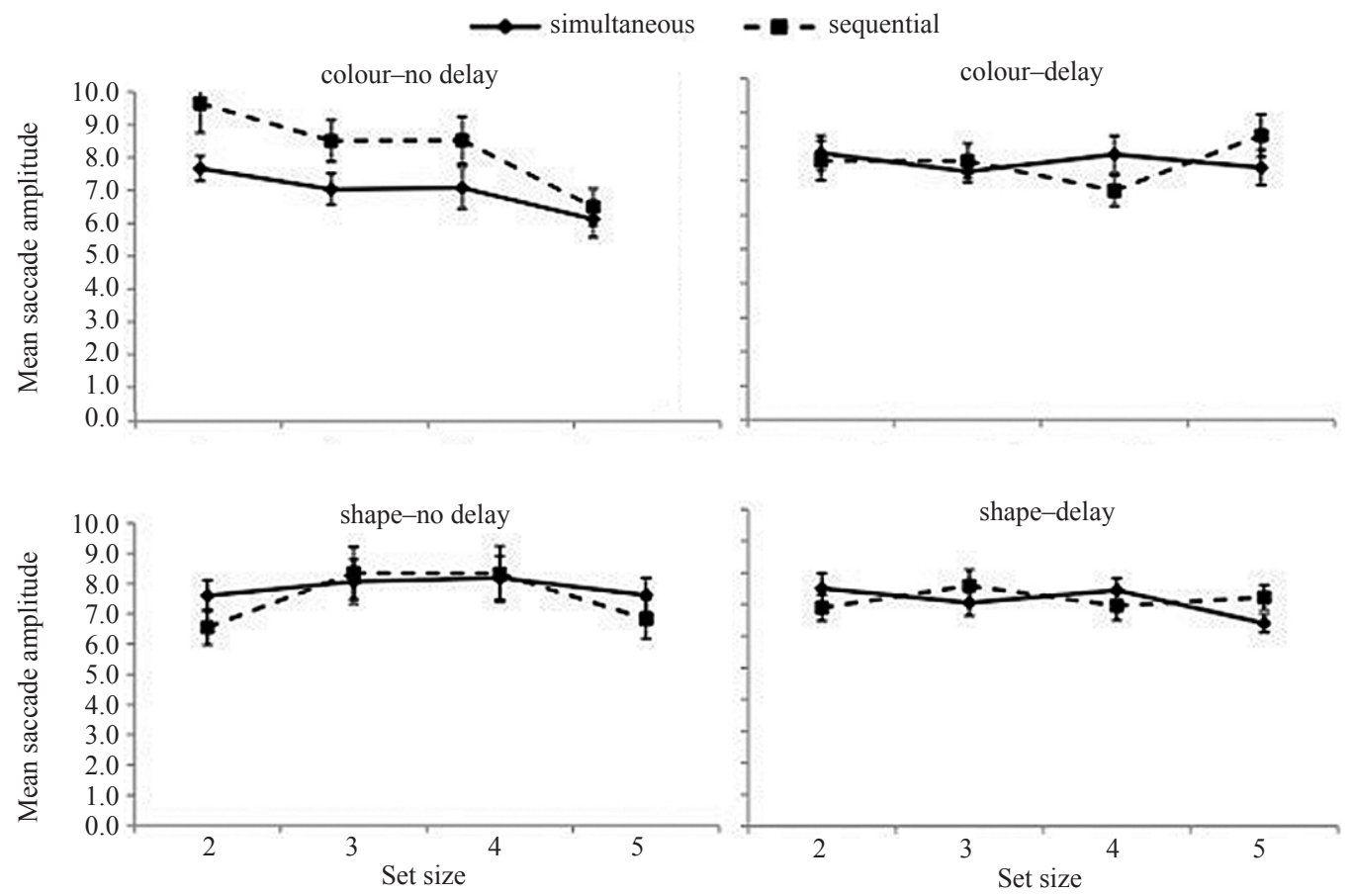

Figure 5. Mean saccade amplitude (deg of visual angle) and standard error during reconstruction as a function of presentation format, feature change, delay, and set size. 


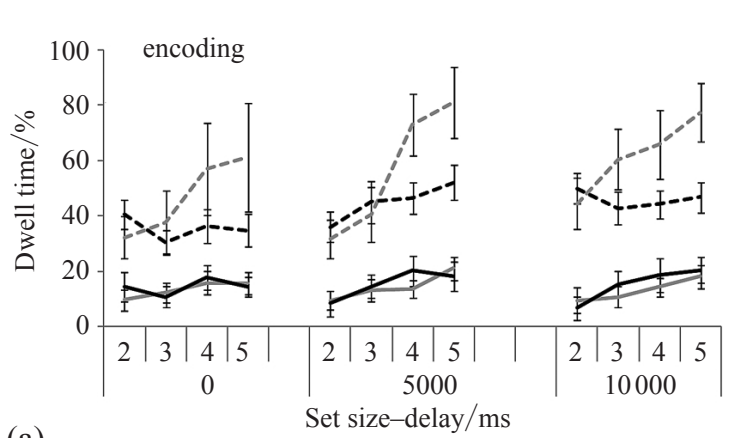

(a)

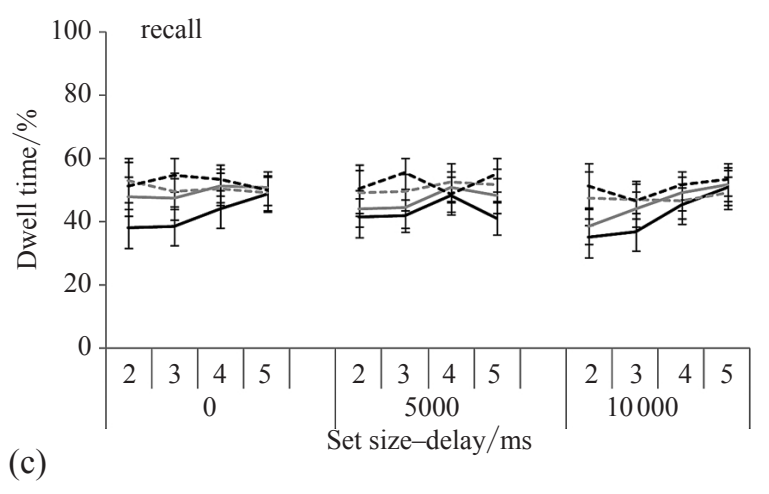

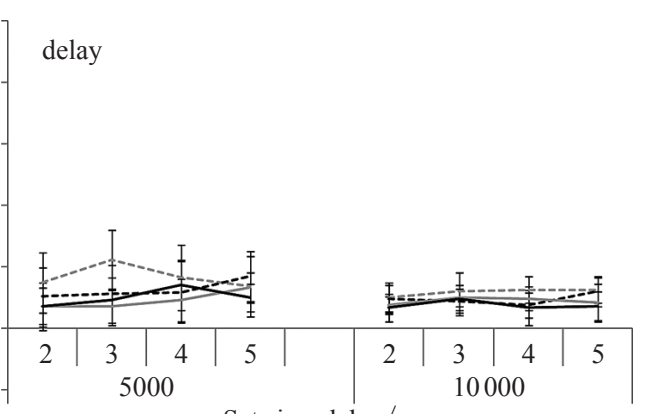

(b)

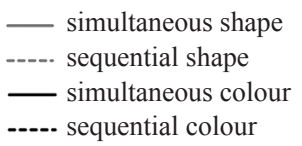

Figure 6. The mean percentage of time the eye has spent looking at the relevant targets within a trial. The graphs show the dwell time (as a \% of total trial time) for fixation within the boundaries of a selected target (ie a target that changed colour or shape) during (a) the encoding stage, (b) the delay, and (c) when recalling the targets. The sequentially presented stimuli are shown as continuous lines, and simultaneous means are shown as dashed lines (grey $=$ shape, black = colour).

\section{Discussion}

The findings of this experiment underline the non-redundancy of eye and recall measures in spatial memory reconstruction. Significant effects of presentation type (sequential versus simultaneous) were found in touch recall accuracy and in eye fixation duration, indicating that participants produced more touch errors and fixated for longer during reconstruction of sequentially presented spatial arrays. In contrast, although the type of feature (colour versus shape) did not affect recall accuracy or reaction time of the hand, we did observe an effect on fixation duration, which on average was longer in colour-change than in shape-change trials. The delay used only showed a significant effect on touch reaction time, with a reduced latency on no delay trials compared to the delay conditions. Finally, set size did affect both touch reaction time and error rate, but had no effect on eye fixation duration. The effects of each of these factors will be discussed in turn.

\subsection{Simultaneous versus sequential presentation}

The higher level of accuracy observed for simultaneous versus sequential presentation conditions is in line with previous findings in visuospatial working memory (eg Allen et al 2006; Blalock and Clegg 2010; Frick 1985; Igel and Harvey 1991; Lecerf and de Ribaupierre 2005; Zimmer et al 2003). For example, Lecerf and de Ribaupierre (2005) found superior recognition accuracy for spatial locations following simultaneous presentation. They suggested that simultaneous presentation allows encoding of the "intrafigural pattern" within an array - that is, the global relationship between items - with this being stored within the visual cache element of Logie's (1995) model of visuospatial working memory (see also 
Zimmer et al 2003). In contrast, sequentially presented spatial locations cannot be easily integrated into a global pattern and are thus solely reliant on spatial memory. The results presented here are in agreement with this suggestion, as our sequentially presented stimuli encouraged longer fixation on each target compared to simultaneous tasks during encoding. We would also suggest that subjects in our task adopt a more central fixation during encoding in simultaneous tasks, which uses a more holistic view of the targets for encoding. These differences may represent one contributory factor involved in the reduced accuracy observed for sequentially presented arrays. It should be noted, however, that these previous experiments used a forced choice same/different button-press response from the participant; therefore accurate reconstruction of the spatial memory was not required and not tested. Our study provides further behavioural evidence of an increase in cognitive effort for the sequential presentation with longer fixation times and greater touch errors.

Sequentially presented sequences also contained temporal order information and indeed require reconstruction of order during the response phase. Connected to this, Frick (1985) suggested that memory for sequentially (but not simultaneously) presented visual stimuli may rely on a timing-based system for storage. The decline in touch accuracy observed in the current study may therefore at least partially reflect problems in implementing or reconstructing this timing signal at recall. Furthermore, we found that eye fixation duration was consistently and significantly longer during reconstruction of sequential spatial patterns. This may indicate additional cognitive demands involved in retrieving order information for each item, thus slowing shifts in spatial attention between locations. Alternatively, it may not be an increased cognitive load that slows fixation shifting but a process of utilising timing information to aid ordered location selection. Thus, as sequentially presented locations were cued at a rate of 1 per second, fixation durations at reconstruction may have slowed to more closely match this temporal rate, which indeed our results reflect. This finding is in-line with previous studies showing motor coordination is largely achieved through learning with timing (Mauk et al 2000) and is mainly achieved via the cerebellum (Miall et al 2001). Shin (2008) used a sequence of random or fixed interval stimuli timings during a serial reaction time task and found no difference between reaction time in the learning of a fixed interval sequence or a random sequence, suggesting that there is no functional benefit from a fixed timing. However, further research would be required to distinguish between these accounts. Nevertheless, the findings from the current study are clear in demonstrating that eye movements slow down when rebuilding a spatial sequence with an order, relative to a simultaneously encountered spatial pattern.

\subsection{Feature change-colour versus shape}

The absence of any effect of feature change on touch accuracy or reaction time would run counter to predictions derived from models of visual attention that emphasise salience (eg Itti and Koch 2001; Koch and Ullman 1985) and links between attention and memory (eg Schmidt et al 2002), as it is generally assumed that colour is more salient than shape (eg Schubö 2009). For example, Fine and Minnery (2009) found that memory for object-location conjunctions were positively correlated with object salience. The lack of error and reaction time differences between colour and shape in the current study would also seem to contradict previous research, showing better memory for colour than shape (eg Allen et al 2006). It is important to note that the current experiment did not require memory for colours or shapes per se but only the locations signalled by these changes.

We found significantly longer fixations on the targets during encoding in the shape task when compared to the colour task, possibly suggesting higher cognitive demands to encode shape stimuli compared to colour. We also observed significantly longer eye fixation durations during reconstruction in trials involving colour change, relative to shape change, indicating 
that change salience (attentional salience) did affect the behavioural response of the eye; however, this was not transmitted downstream into the response of the hand. Evidence exists which suggests that longer fixation times result in better recall performance (Holingworth and Henderson 2002). Fixation duration in this case indicates the extent to which participants visually scanned the array before making each touch response. The reduced salience of shape changes may have led participants to perform more scanning of the display during the reconstruction phase, in an effort to reconstruct the spatial patterns that they represented. This would manifest in shorter eye-fixation times relative to colour trials but overall similar fixations on relevant targets as observed in the recall ROI analysis. Thus, these findings fit with the view that spatial attention and spatial working memory may both be connected to the oculomotor system (eg Curtis 2006; Pearson and Sahraie 2003; Theeuwes et al 2009). This explanation also fits with the significant interaction we observed between feature change and delay, in that colour change trials showed longer fixations only in no delay trials, while there was minimal difference between colour and shape trials following retention intervals of $5 \mathrm{~s}$ or $10 \mathrm{~s}$. The particular salience of colour changes may only reduce the need for increased reconstruction-based scanning over little or no retention intervals, presumably because such changes initially remain salient in memory. However, when delays are longer, the relatively greater salience of colour than shape changes diminishes, and all feature-change trials require equivalent levels of rescanning in order to reconstruct spatial arrays. These findings are also noteworthy in demonstrating how eye movement data can reveal processes that are not detectable through the use of standard button press recall measures.

\subsection{Delay}

Delay duration significantly influenced only touch reaction time, with reduced latency on trials with a retention interval (blank screen) between presentation offset and the response phase. This may reflect a delay in participants reacting to the cue to respond after these longer intervals, rather than any meaningful decrease in memory processes. This is supported by the observation that no significant decrement in touch accuracy was observed between any of the delay durations. This would seem to contrast with the classic work of Phillips (1974), who found that visual pattern memory did decline over retention intervals up to $9 \mathrm{~s}$. However, in a more recent study, Vogel et al (2001) did not observe any decrement in accuracy for colours over delays up to $5 \mathrm{~s}$. It should be noted that these studies are more closely associated with visual than spatial memory. Dreher et al (2001) examined memory for spatial locations using touch reconstruction (with and without order information, among other conditions) and, in line with our findings, did not observe significant accuracy differences between $500 \mathrm{~ms}$ and $10 \mathrm{~s}$ delay effects in healthy controls, although they did find delay effects in patients with schizophrenia, possibly indicating something of a role for attentional control in keeping representations active in working memory. In line with this, participants in our study reported that they engaged in rehearsal of locations during the retention intervals (see Awh et al 1998; Pearson and Sahraie 2003; Theeuwes et al 2009).

We also did not observe an effect of delay on eye-fixation duration during the reconstruction phase. Thus, while presentation type (including order information and change salience) does influence the degree of scanning that takes place at reconstruction, delay does not have an effect. This may be due to rehearsal during retention minimising the impact of these delays. This suggestion may lead to the prediction that ocular suppression (eg Guérard et al 2009; Pearson and Sahraie 2003) would have an effect on both touch accuracy and eye-fixation duration, a possibility that could be tested in future research. 


\subsection{Set size}

We found that, overall, touch accuracy declined with increasing set size, supporting the common conception of spatial working memory as a limited capacity system. Indeed, in the typical Corsi span task (on which our measures are closely based), set size is increased for a given participant until incorrect responses begin to emerge (Corsi 1972). It is worth noting that, as our primary aim was to examine eye-fixation duration during recall of sub-span and span-length sequences, the set sizes used in this experiment were typically within or at the limit of spatial memory capacity (typically, around 5 items in Corsi span). Nevertheless, we were still able to detect a small decline in accuracy (see also Dreher et al 2001).

Touch accuracy and reaction time declined with increasing set size; thus participants responded faster for larger item sets (counter to our initial prediction). This contrasts with the findings of Magen et al (2009), who found increasing reaction time with set size in a visuospatial memory task. However, their study used a yes/no recognition task with a single key press response, which likely involves very different decision processes from touch-based spatial reconstruction. The faster reaction times for larger spatial arrays that were observed with this measure may simply indicate that participants felt more motivated to reconstruct these displays more quickly, to minimise effects of forgetting, which is more significant with a greater number of items.

As with the delay factor, we did not find an effect of set size on eye-fixation duration during recall. This result could be explained by assuming that scanning of the visual array at test occurs for each individual item; however, scanning at recall is no longer required, and therefore the same extent of scanning would occur across different set sizes and would not increase as a function of how many items need to be reconstructed. Indeed, we found that subjects make on average 2-3 saccades during the reconstruction phase of the response regardless of the number of items, delay, or condition. Finally, the increasing dwell time on the selected target items with increasing set size in the sequentially presented shape tasks but not in other conditions also provides further evidence of attentional differences in encoding between colour and shape.

\section{Conclusion}

A number of previous studies have used a forced-choice button press response to look at the affects of sequential versus simultaneous presentation types on short-term memory performance. These previous experiments are limited in understanding how behaviour of the eye and hand influence and/or contribute to the overall response, and they use only reaction time. The current experiment used a free-viewing sequential versus simultaneous spatial memory task in order to establish whether the accuracy of a behavioural response is influenced by attention and memory in a similar way to reaction time.

We found increasing the number of items in a task and needing to remember the order of the items produced more spatial errors in the touch responses than did remembering the location alone. This demonstrates that additional storage resources are required for both remembering the location and order rather than location alone and further suggests these processes are achieved separately in the brain. The eye movement analysis revealed longer fixation times for sequentially presented locations during reconstruction, possibly reflecting an attempt by participants to mimic the original timing of target onsets, which may serve to enhance recall. The delay had no effect on the accuracy of locating and remembering the order of the targets; however, subjects were quicker to respond after a delay indicating a motor preparedness rather than a cognitive decline after a delay. Overall, this study shows that the type of information (ie location and/or temporal order) and number of items have significant effects on motor behaviour and memory when generating a correct touch response. 
In contrast, manipulation of the attentional salience of target cueing (ie colour or shape) tends to affect the eye movement but has little effect on how well this information is retained for the touch response.

\section{References}

Allen R J, Baddeley A D, Hitch G J, 2006 "Is the binding of visual features in working memory resource-demanding?" Journal of Experimental Psychology: General 135 298-313

Awh E, Jonides J, 2001 "Overlapping mechanisms of attention and spatial working memory" Trends in Cognitive Sciences 5 119-126

Awh E, Jonides J, Reuter-Lorenz P, 1998 "Rehearsal in spatial working memory" Journal of Experimental Psychology: Human Perception and Performance 24 780-790

Awh E, Vogel E K, Oh S, 2006 "Interactions between attention and working memory" Neuroscience 139 201-208

Baddeley A D, 1986 Working Memory (Oxford, UK: Oxford University Press)

Baddeley A D, 2003 "Working memory: looking back and looking forward" Nature Reviews Neuroscience 4 829-839

Baddeley A D, Hitch G J, 1974 "Working memory", in The Psychology of Learning and Motivation Ed. G H Bower (New York: Academic Publisher) pp 47-89

Belopolsky A V, Theeuwes J, 2009 "When are attention and saccade preparation dissociated?" Psychological Science 20 1340-1347

Blalock L D, Clegg B A, 2010 "Encoding and representation of simultaneous and sequential arrays in visuospatial working memory" Quarterly Journal of Experimental Psychology 63 856-862

Chan L, Hayward W, Theeuwes J, 2009 "Spatial working memory maintenance: Does attention play a role? A visual search study" Acta Psychologica 132 115-123

Corsi P M, 1972 "Human memory and the medial temporal region of the brain" Dissertation Abstracts International 34 891B

Courtney S M, Ungerleider L G, Keil K, Haxby J V, 1996 “Object and spatial visual working memory activate separate neural systems in human cortex" Cerebral Cortex 6 39-49

Cowan N, 2001 "The magical number 4 in short-term memory: A reconsideration of mental storage capacity" Behavioral and Brain Sciences 24 87-114

Curtis C E, 2006 "Prefrontal and parietal contributions to spatial working memory" Neuroscience 139 $173-180$

Della Sala S, Gray C, Baddeley A D, Allamano N, Wilson L, 1999 "Pattern span: a tool for unwelding visuo-spatial memory" Neuropsychologia 37 1189-1199

De Renzi E, Nichelli P, 1975 "Verbal and non-verbal short-term memory impairment following hemispheric damage" Cortex 11 341-353

D’Esposito M, Detre J, Alsop D, Shin R, Atlas S, Grossman M, 1995 "The neural basis of the central executive system of working memory" Nature 378 279-281

Dreher J C, Banquet J P, Allilaire J F, Paillère-Martinot M L, Dubois B, Burnod Y, 2001 "Temporal order and spatial memory in schizophrenia: a parametric study" Schizophrenia Research $\mathbf{5 1}$ $137-147$

Eimer M, 1997 "An event-related potential (ERP) study of transient and sustained visual attention to color and form" Biological Psychology 44 143-160

Elliot R, Dolan R, 1999 "Differential neural responses during performance of matching and nonmatching to sample tasks at two delay intervals" Journal of Neuroscience 19 5066-5073

Fecteau J, Munoz D, 2006 "Salience, relevance, and firing: a priority map for target selection" Trends in Cognitive Sciences 10 382-390

Fine M, Minnery B, 2009 "Visual salience affects performance in a working memory task" Journal of Neuroscience 29 8016-8021

Frick R, 1985 "Testing visual short-term memory: simultaneous versus sequential presentations" Memory and Cognition 13 346-356

Grossi D, Becker J T, Smith C, Trojano L, 1993 “Memory for visuo-spatial patterns in Alzheimer's disease" Psychological Medicine 23 63-70 
Guérard K, Neath I, Surprenant A M, Tremblay S, 2010 "Distinctiveness in serial memory for spatial information" Memory and Cognition 38 83-91

Guérard K, Tremblay S, Saint-Aubin J, 2009 "The processing of spatial information in short-term memory: insights from eye tracking the path length effect" Acta Psychologica 132 136-144

Hollingworth A, Henderson J M, 2002 "Accurate visual memory for previously attended objects in natural scenes" Journal of Experimental Psychology: Human Perception and Performance 28 $113-136$

Igel A, Harvey L O, 1991 "Spatial distortions in visual perception" Gestalt Theory 13 210-231

Itti L, Koch C, 2001 "Computational modeling of visual attention" Nature Reviews Neuroscience 2 194-203

Joyce E M, Robbins T W, 1991 "Frontal lobe function in Korsakoff and non-Korsakoff alcoholics: planning and spatial working memory" Neuropsychologia 29 709-723

Klauer K C, Zhao Z, 2004 "Double dissociations in visual and spatial short-term memory" Journal of Experimental Psychology: General 133 355-381

Koch C, Ullman S, 1985 "Shifts in selective visual attention: towards the underlying neural circuitry" Human Neurobiology 4 219-227

Lecerf T, de Ribaupierre A, 2005 "Recognition in a visuospatial memory task: the effect of presentation" European Journal of Cognitive Psychology 17 47-75

Linden D, Bittner R, Muckli L, Waltz J, Kriegeskorte N, Goebel R, Singer W, Munk M, 2003 “Cortical capacity constraints for visual working memory: dissociation of fMRI load effects in a frontoparietal network" NeuroImage 20 1518-1530

Logie R H, 1995 Visuo-Spatial Working Memory (Hove, UK: Lawrence Erlbaum Associates)

Luck S J, Vogel E K, 1997 "The capacity of visual working memory for features and conjunctions" Nature 390 279-281

Magen H, Emmanouil T-A, McMains S, Kastner S, Treisman A, 2009 "Attentional demands predict short-term memory load response in posterior parietal cortex" Neuropsychologia 47 1790-1798

Mangels J A, 1997 "Strategic processing and memory for temporal order in patients with frontal lobe lesions" Neuropsychology 11 207-221

Marshuetz C, Smith E, Jonides J, DeGutis J, Chenevert T, 2000 “'Order information in working memory: fMRI evidence for parietal and prefrontal mechanisms" Journal of Cognitive Neuroscience 12 $130-144$

Miall R C, Reckess G Z, 2002 "The cerebellum and the timing of coordinated eye and hand tracking" Brain and Cognition 48 212-226

Miall R C, Reckess G Z, Imamizu H, 2001 "The cerebellum coordinates eye and hand tracking movements" Nature Neuroscience 4 638-644

Milner B, 1971 "Interhemispheric differences in the localization of psychological processes in man" British Medical Bulletin 27 272-277

Müller N G, Knight R T, 2006 "The functional neuroanatomy of working memory: contributions of human brain lesion studies" Neuroscience 139 51-58

Pearson D, Sahraie A, 2003 "Oculomotor control and the maintenance of spatially and temporally distributed events in visuo-spatial working memory" Quarterly Journal of Experimental Psychology A 56 1089-1111

Phillips W, 1974 "On the distinction between sensory storage and short-term visual memory" Perception \& Psychophysics 16 283-290

Posner M, 1980 "Orienting of attention: The 12th Bartlett Memorial Lecture" Quarterly Journal of Experimental Psychology 32 3-25

Proverbio A, Burco F, del Zotto M, Zani A, 2004 "Blue piglets? Electrophysiological evidence for the primacy of shape over color in object recognition" Cognitive Brain Research 18 288-300

Saint-Aubin J, Tremblay S, Jalbert A, 2007 "Eye movements and serial memory for visual-spatial information: does time spent fixating contribute to recall?" Experimental Psychology 54 264-272

Sakai K, Passingham R E, 2003 "Prefrontal interactions reflect future task operations" Nature Neuroscience $\mathbf{6} 75-81$

Schmidt B K, Vogel E K, Woodman G F, Luck S J, 2002 "Voluntary and automatic attentional control of visual working memory” Perceptual Psychophysics 64 754-763 
Schubö A, 2009 "Salience detection and attentional capture" Psychological Research 73 233-243

Shah P, Miyake A, 1999 "Models of working memory", in Models of Working Memory: Mechanisms of Active Maintenance and Executive Control Eds A Miyake, P Shah (Cambridge, UK: Cambridge University Press) pp 1-27

Shin J, 2008 "The procedural learning of action order is independent of temporal learning" Psychological Research 72 376-386

Smyth M M, 1996 "Interference with rehearsal in spatial working memory in the absence of eye movements" Quarterly Journal of Experimental Psychology A 49 940-949

Smyth M M, Scholey K A, 1994 "Interference in immediate spatial memory" Memory and Cognition $221-13$

Theeuwes J, Belopolsky A, Olivers C N, 2009 "Interactions between working memory, attention and eye movements" Acta Psychologia 132 106-114

Todd J J, Marois R, 2005 "Posterior parietal cortex activity predicts individual differences in visual short-term memory capacity" Cognitive, Affective, \& Behavioral Neuroscience 2 144-155

Treisman A M, Gelade G, 1980 "A feature-integration theory of attention" Cognitive Psychology 12 $97-136$

Treisman A, Zhang W, 2006 "Location and binding in visual working memory" Memory and Cognition 34 1704-1719

Tremblay S, Saint-Aubin J, Jalbert A, 2006 "Rehearsal in serial memory for visual-spatial information: evidence from eye movements" Psychonomic Bulletin \& Review 13 452-457

Tresch M C, Sinnamon H M, Seamon J G, 1993 "Double dissociation of spatial and object visual memory: evidence from selective interference in intact human subjects" Neuropsychologia 31 211-219

Uchikawa K, Shinoda K, 1996 "Influence of basic color categories on color memory discrimination" Color Research and Application 21 430-439

Vandierendonck A, Kemps E, Fastame M C, Szmalec A, 2004 "Working memory components of the Corsi blocks task" British Journal of Psychology 95 57-79

Vogel E, Woodman G, Luck S, 2001 "Storage of features, conjunctions, and objects in visual working memory" Journal of Experimental Psychology: Human Perception and Performance 27 92-114

Vogel E, Woodman G, Luck S, 2006 "Time course of consolidation in visual working memory" Journal of Experimental Psychology: Human Perception and Performance 32 1436-1451

Zhang B, Zhang J X, Kong L, Huang S, Yue Z, Wang S, 2010 "Guidance of visual attention from working memory contents depends on stimulus attributes" Neuroscience Letters 486 202-206

Zimmer H D, Speiser H R, Seidler B, 2003 "Spatio-temporal working memory and short-term object location tasks use different memory mechanisms" Acta Psychologica 114 41-65 